\title{
The degree of approximation by Hausdorff means of a conjugate Fourier series
}

\begin{abstract}
The purpose of this paper is to analyze the degree of approximation of a function $\bar{f}$ that is a conjugate of a function $f$ belonging to the Lipschitz class by Hausdorff means of a conjugate series of the Fourier series.
\end{abstract}

1. Introduction. The sequence of partial sums $s_{n}(x)$ for a conjugate series of the Fourier series of function $f$ converges at the point $x$ to the number $\bar{f}(x)=-\frac{1}{2 \pi} \int_{0}^{\pi} \frac{f(x+t)-f(x-t)}{\tan (t / 2)} d t$ if the function $f$ at the point $x$ satisfies the Lipschitz condition $|f(x \pm t)-f(x)|<C t^{\alpha}$ for $\alpha \in(0,1]$. The main focus of our analysis relates to the speed with which the mentioned instance occurs. If we assume the degree of approximation $\sup _{x \in R}\left|s_{n}(x)-\bar{f}(x)\right|$, then the following question arises: does the sequence $(n+1)^{\alpha} \sup _{x \in R}\left|s_{n}(x)-\bar{f}(x)\right|$ continue to be a bounded sequence? Our analysis is conducted using certain regular methods.

Several studies have been conducted on the degree of approximation of a function by different summability means of its Fourier series. For the first time in 1981, Qureshi [9] discussed the degree of approximation of the conjugate of a function belonging to a Lipschitz class by Nörlund means of conjugate Fourier series. He proved the following:

2010 Mathematics Subject Classification. Primary 40G05; Secondary 42A50.

Key words and phrases. Hausdorff matrix, conjugate series of the Fourier series, degree of approximation. 
Theorem 1.1. Let $f$ be a $2 \pi$-periodic function and Lebesgue integrable and let

$$
\bar{f}(x)=\sum_{n=1}^{\infty}\left(b_{n} \cos n x-a_{n} \sin n x\right) .
$$

Let $\left\{P_{n}\right\}$ be a sequence of positive constants such that $P_{n}=p_{0}+\ldots+p_{n} \rightarrow \infty$ if $n \rightarrow \infty$ and $t_{n}(x)=\left(p_{n} s_{0}(x)+p_{n-1} s_{1}(x)+\ldots+p_{0} s_{n}(x)\right) / P_{n}$. If $\left\{P_{n}\right\}$ satisfies the conditions $n\left|p_{n}\right|<\left|P_{n}\right|$ and $\sum_{k=1}^{n} k\left|p_{k}-p_{k-1}\right|<C\left|P_{n}\right|$, then the degree of approximation of the function $\bar{f}(x)$ is given by $\left|\bar{f}(x)-t_{n}(x)\right|=$ $O\left(\frac{1}{P_{n}} \sum_{k=1}^{n} \frac{p_{k}}{k^{1+\alpha}}\right)$, where $f \in \operatorname{Lip} \alpha$ for $\alpha \in(0,1]$ and $\left\{s_{n}(x)\right\}$ is the sequence of partial sums of a conjugate series of the Fourier series of $f$.

The problem of the degree of approximation of a function belonging to $\operatorname{Lip} \alpha$ was the subject of many studies, including the recently published works of Lal and Mishra [6], [7].

In the first part of this paper, we formulate and prove the variant of the theorem discussed by Lal [6]. The obtained results (Theorem 2.1) will be applied in the analysis of the degree of approximation for certain Hausdorff methods. To the best of our knowledge, no one has investigated the speed of convergence of the Hölder summation method. Theorem 2.7 of the Hölder method is the first remarkable result of our work based on Theorem 2.1.

In the second part of this paper, we prove that the generalization of the obtained results, with respect to the entire class of Hausdorff methods of a conjugate Fourier series, is impossible. This result, as the second instrumental finding of our research, is interesting because the generalization with respect to the entire class of Hausdorff methods of a Fourier series is possible (cf. [10]). A counterexample formulated as Theorem 2.8 proves that the hypothesis presented in the work by Lal and Mishra [7] is false when the parameter of $\alpha$ is limited to the range $\left(0, \frac{1}{2}\right]$.

An infinite matrix $C=\left[c_{m n}\right], m, n=0,1, \ldots$ is called a regular matrix (method) if it transforms any convergent sequence into a convergent sequence with the same limit.

In 1911, Toeplitz presented the following equivalent conditions for regularity [11].

Theorem 1.2. The matrix $C=\left[c_{m n}\right]$ is regular if and only if

T1) $\forall n \geq 0 \lim _{m \rightarrow \infty} c_{m n}=0$;

T2) $\quad \lim _{m \rightarrow \infty} \sum_{n=0}^{\infty} c_{m n}=1$;

T3) $\exists M>0 \forall m \geq 0 \sum_{n=0}^{\infty}\left|c_{m n}\right|<M$.

In 1921, Hausdorff [2] proved the following: 
Theorem 1.3. Given the sequence $\left(\mu_{n}\right)_{n=1}^{\infty}$, define

$$
\Delta^{p} \mu_{n}=\sum_{i=0}^{p}\left(\begin{array}{c}
p \\
i
\end{array}\right)(-1)^{i} \mu_{n+i} .
$$

Then the matrix $\Lambda$ with elements

$$
\lambda_{m n}= \begin{cases}\left(\begin{array}{l}
m \\
n
\end{array}\right) \Delta^{m-n} \mu_{n} & \text { for } n \leq m, \\
0 & \text { for } n>m\end{cases}
$$

is regular if and only if $\mu_{n}$ is the moment sequence

$$
\mu_{n}=\int_{0}^{1} x^{n} d \chi(x)
$$

where $\chi$ is a real, bounded variation function defined on the interval $[0,1]$ satisfying the conditions

$$
\chi(0+)=\chi(0)=0 \quad \text { and } \quad \chi(1)=1 .
$$

A sequence $\mu_{n}$ that satisfies the conditions (1.2) is known as a moment sequence, while a sequence that satisfies both conditions (1.2) and (1.3) is known as a Hausdorff moment sequence. The matrix $\Lambda$ in (1.1) that satisfies both (1.2) and (1.3) is known as a Hausdorff matrix (method). A real sequence $s_{n}$ is considered to be summable by the Hausdorff matrix (method) if there exists a function $\chi$ that satisfies the conditions (1.2) and (1.3) so that the sequence

$$
v_{m}=\sum_{n=0}^{\infty} \lambda_{m n} s_{n}
$$

with $\lambda_{m, n}$ defined as in (1.1) is convergent. For the proof of the above theorems, see the work by Hardy [1].

Since 1921, the Hausdorff theorem has been subject to numerous studies and generalizations. In 1933, the theorem was proved within the framework of three-index matrices by Hildebrandt and Schoenberg [3]. Kęska [5] proved the theorem for multi-index Hausdorff matrices. Jakimovski [4] presented a variant of the Hausdorff theorem that extended to the space of sequences of functions.

Let $f$ be a $2 \pi$-periodic function and Lebesgue integrable on $[-\pi, \pi]$. The Fourier series of $f$ at a point $x$ is defined by

$$
f(x) \sim \frac{a_{0}}{2}+\sum_{n=0}^{\infty}\left(a_{n} \cos n x+b_{n} \sin n x\right),
$$

where $a_{n}=\frac{1}{\pi} \int_{-\pi}^{\pi} f(u) \cos n u d u$ and $b_{n}=\frac{1}{\pi} \int_{-\pi}^{\pi} f(u) \sin n u d u$. 
The conjugate series of a Fourier series (1.5) of $f$ is given by

$$
\sum_{n=0}^{\infty}\left(a_{n} \sin n x-b_{n} \cos n x\right),
$$

with $n$-th partial sums $s_{n}(x)$.

The degree of approximation of a function $f: R \rightarrow R$ by a trigonometric polynomial $p_{n}(x)$ of order $n$ is defined by

$$
\left\|p_{n}-f\right\|_{\infty}=\sup _{x \in R}\left|p_{n}(x)-f(x)\right| .
$$

In 2004, Lal [6] proved the following theorem:

Theorem 1.4. Let $T=\left(a_{n k}\right)$ be a lower triangular matrix with finite norms and

$$
\sum_{k=0}^{n} \frac{a_{n k}}{k+1}=O\left(\frac{1}{n+1}\right) .
$$

If $f: R \rightarrow R$ is a $2 \pi$ periodic function, Lebesgue integrable on $[-\pi, \pi]$ and almost $\operatorname{Lip} \alpha$ (there is a constant $M_{f}>0$ and $\forall x \in R$ there is a subset $A_{x} \subset$ $[0, \pi / 2]$ of measure zero such that $t \in[0, \pi / 2]-A_{x}$ implies $\mid f(x+2 t)-f(x-$ $\left.2 t) \mid=M_{f} t^{\alpha}\right)$, then the degree of approximation of its conjugate function $\bar{f}$ by matrix Cesàro product means $v_{n}=\sum_{k=0}^{n} a_{n k} \sigma_{k}$ of the conjugate series (1.6) satisfies, for $n=0,1,2, \ldots$,

$$
\left\|v_{n}-\bar{f}\right\|_{\infty}= \begin{cases}O\left(\frac{1}{(n+1)^{\alpha}}\right) & \text { for } \alpha<1 \\ O\left(\frac{\log (n+1)}{n+1}\right) & \text { for } \alpha=1\end{cases}
$$

where

$$
\sigma_{k}=\frac{1}{k+1} \sum_{r=0}^{k} s_{r}, \quad s_{r}(x)-\bar{f}=(1 / \pi) \int_{0}^{\pi / 2} \frac{\cos (2 r+1) t d t}{\sin t},
$$

$\bar{f}(x)=(-1 / \pi) \int_{0}^{\pi / 2} \Psi(t) \cot t d t$ and $\Psi(t)=f(x+2 t)-f(x-2 t)$.

\section{The degree of approximation by Hausdorff means of a conju-} gate Fourier series. We first formulate a variant of Theorem 1.4.

Theorem 2.1. Let $f$ be a $2 \pi$-periodic function and Lebesgue integrable on $[-\pi, \pi]$, which satisfies the Lipschitz condition for $0<\alpha<1$

$$
|f(x \pm t)-f(x)|=O\left(t^{\alpha}\right) \forall t \in[0, \pi], x \in R .
$$

Let $\Psi_{x}(t)=f(x+t)-f(x-t), A=\left[c_{n k}\right]$ be a regular matrix, which satisfies the condition

$$
\sum_{k=0}^{n} \frac{\left|c_{n k}\right|}{(k+1)^{\alpha}}=O\left(\frac{1}{(n+1)^{\alpha}}\right)
$$


Let $C^{1}$ denote the Cesàro method of order 1 . Let $s_{n}(x)$ denote the $n$-th partial sum of conjugate series of the Fourier series of $f$. Then for $0<\alpha<$ 1 we obtain

$$
\left\|A C^{1}\left(s_{n}\right)-\bar{f}\right\|_{\infty}=O\left(\frac{1}{(n+1)^{\alpha}}\right)
$$

where

$$
\bar{f}(x)=-\frac{1}{2 \pi} \int_{0}^{\pi} \Psi_{x}(t) \frac{d t}{\tan (t / 2)} .
$$

Proof. By virtue of results of [8] and based on the assertion in (2.1) we may conclude that

$$
\left|a_{n}(x)\right|=\left|C^{1}\left(s_{n}(x)\right)-\bar{f}(x)\right| \leq \frac{G}{(n+1)^{\alpha}} .
$$

Let

$$
b_{m}(x)=A\left(C^{1}\left(s_{n}(x)\right)\right)-\bar{f}(x)=A\left(C^{1}\left(s_{n}(x)\right)-\bar{f}(x)\right)=\sum_{n=0}^{m} c_{m n} a_{n}(x),
$$

where $A=\left[c_{m n}\right]$. Then $(m+1)^{\alpha} b_{m}(x)=(m+1)^{\alpha} \sum_{n=0}^{m} c_{m n} a_{n}(x)$ and

$$
\begin{aligned}
\left|(m+1)^{\alpha} b_{m}(x)\right| & \leq(m+1)^{\alpha} \sum_{n=0}^{m}\left|c_{m n}\right|\left|a_{n}(x)\right| \\
& \leq G \sum_{n=0}^{m}\left|c_{m n}\right| \frac{(m+1)^{\alpha}}{(n+1)^{\alpha}} \leq G_{1} .
\end{aligned}
$$

This is a consequence of (2.2).

Definition 2.2. A moment sequence $\mu_{n}$ is known as a right-shifting sequence of moments if there exists $\mu_{0}^{*} \in R$ such that the sequence $\left(\mu_{0}^{*}, \mu_{1}^{*}=\right.$ $\left.\mu_{0}, \ldots, \mu_{k+1}^{*}=\mu_{k}, \ldots\right)$ is a moment sequence $(1.2)$.

Corollary 2.3. A matrix $\Lambda=\left[\lambda_{n k}\right]$, where $\lambda_{n k}=\left(\begin{array}{l}n \\ k\end{array}\right) \Delta^{n-k} \mu_{k}$ for $n \geq k \geq$ 0 and 0 for others, generated on the basis of a right-shifting sequence of moments $\mu_{n}$, fulfils the condition (2.2).

Proof. We have

$$
(n+1)^{\alpha} \sum_{k=0}^{n}\left|\frac{\lambda_{n k}}{(k+1)^{\alpha}}\right|=\frac{1}{(n+1)^{1-\alpha}} \sum_{k=0}^{n}(k+1)^{1-\alpha}\left(\begin{array}{c}
n+1 \\
k+1
\end{array}\right)\left|\Delta^{n-k} \mu_{k}\right| .
$$

We know that the sequence $\left(\mu_{0}^{*}, \mu_{1}^{*}=\mu_{0}, \ldots, \mu_{k+1}^{*}=\mu_{k}, \ldots\right)$ is a moment sequence. Applying Theorem 204 [1], we obtain

$$
\mu_{l}^{*}=\mu_{l}^{*+}-\mu_{l}^{*-}, \text { where } \forall l, p \geq 0 \Delta^{p} \mu_{l}^{* \pm} \geq 0 .
$$


Then,

$$
\begin{aligned}
(n+1)^{\alpha} \sum_{k=0}^{n}\left|\frac{\lambda_{n k}}{(k+1)^{\alpha}}\right| & =\frac{1}{(n+1)^{1-\alpha}} \sum_{k=0}^{n}(k+1)^{1-\alpha}\left(\begin{array}{c}
n+1 \\
k+1
\end{array}\right)\left|\Delta^{n-k} \mu_{k+1}^{*}\right| \\
\leq & \frac{1}{(n+1)^{1-\alpha}} \sum_{k=0}^{n}(k+1)^{1-\alpha}\left(\begin{array}{c}
n+1 \\
k+1
\end{array}\right) \Delta^{n-k} \mu_{k+1}^{*+} \\
& +\frac{1}{(n+1)^{1-\alpha}} \sum_{k=0}^{n}(k+1)^{1-\alpha}\left(\begin{array}{c}
n+1 \\
k+1
\end{array}\right) \Delta^{n-k} \mu_{k+1}^{*-} \\
\leq & \sum_{l=1}^{n+1}\left(\begin{array}{c}
n+1 \\
l
\end{array}\right) \Delta^{n+1-l} \mu_{l}^{*+}+\sum_{l=1}^{n+1}\left(\begin{array}{c}
n+1 \\
l
\end{array}\right) \Delta^{n+1-l} \mu_{l}^{*-} \\
= & \sum_{l=0}^{n+1}\left(\begin{array}{c}
n+1 \\
l
\end{array}\right) \Delta^{n+1-l} \mu_{l}^{*+}+\sum_{l=0}^{n+1}\left(\begin{array}{c}
n+1 \\
l
\end{array}\right) \Delta^{n+1-l} \mu_{l}^{*-} \\
& -\Delta^{n+1} \mu_{0}^{*+}-\Delta^{n+1} \mu_{0}^{*-} .
\end{aligned}
$$

After considering

$$
\sum_{l=0}^{n+1}\left(\begin{array}{c}
n+1 \\
l
\end{array}\right) \Delta^{n+1-l} \mu_{l}^{* \pm}=\mu_{0}^{* \pm}
$$

(see p. $252[1]$ ) and $\Delta^{n+1} \mu_{0}^{* \pm} \geq 0$ (which follows from (2.7)), we obtain $(n+1)^{\alpha} \sum_{k=0}^{n}\left|\frac{\lambda_{n k}}{(k+1)^{\alpha}}\right| \leq H$.

Example 1. The Hausdorff moment sequence $\mu_{n}=\frac{1}{(q+1)^{n}}$, which generates the Euler method $(E, q)$, is a right-shifting sequence of moments because $\Delta^{n} \mu_{0}^{*}=(1+q) \Delta^{n} \mu_{0} \geq 0$ for $\mu_{0}^{*}=(1+q)$.

Example 2. The Hausdorff moment sequence $\mu_{n}=\frac{1}{(n+1)}$, which generates the Cesàro method $C^{1}$, is not a right-shifting sequence of moments.

Proof. If $\mu_{n}$ is a right-shifting sequence of moments, then there exists such a $\mu_{0}^{*}$ that makes the sequence $\left(\mu_{0}^{*}, \mu_{1}^{*}=\mu_{0}, \ldots, \mu_{k+1}^{*}=\mu_{k}, \ldots\right)$ a sequence of moments. It is important to observe that the sequences $\Delta^{n} \mu_{0}^{* \pm}$ must be positive and decreasing with $n$, which follows from the relationship $\Delta^{n+1} \mu_{0}^{* \pm}-\Delta^{n+2} \mu_{0}^{* \pm}=\Delta^{n+1} \mu_{1}^{* \pm}$ and (2.7). Moreover,

$$
-\sum_{i=1}^{n}(-1)^{i}\left(\begin{array}{c}
n \\
i
\end{array}\right) 1 / i=\sum_{i=1}^{n} 1 / i,
$$


which follows from $\left(\begin{array}{c}n+1 \\ i\end{array}\right)=\left(\begin{array}{c}n \\ i\end{array}\right)+\left(\begin{array}{c}n \\ i-1\end{array}\right)$ and $\left(\begin{array}{c}n \\ i-1\end{array}\right) \frac{1}{i}=\left(\begin{array}{c}n+1 \\ i\end{array}\right) \frac{1}{n+1}$. After considering (2.8), we can calculate

$$
\begin{aligned}
\Delta^{n} \mu_{0}^{*} & =\sum_{i=0}^{n}(-1)^{i}\left(\begin{array}{c}
n \\
i
\end{array}\right) \mu_{i}^{*}=\mu_{0}^{*}+\sum_{i=1}^{n}(-1)^{i}\left(\begin{array}{c}
n \\
i
\end{array}\right) \mu_{i-1} \\
& =\mu_{0}^{*}+\sum_{i=1}^{n}(-1)^{i}\left(\begin{array}{c}
n \\
i
\end{array}\right) 1 / i=\mu_{0}^{*}-\sum_{i=1}^{n} 1 / i .
\end{aligned}
$$

Thus, the sequence $\Delta^{n} \mu_{0}^{*}$ cannot be convergent, which is a contradiction.

Remark 2.4. However, the Cesàro method $C^{1}$ satisfies condition (2.2).

\section{Proof.}

$$
\begin{aligned}
& \lim _{n \rightarrow \infty} \frac{(n+1)^{\alpha}}{n+1} \sum_{k=0}^{n} 1 /(k+1)^{\alpha}=\lim _{n \rightarrow \infty} \frac{1 /(n+1)^{\alpha}}{(n+1)^{1-\alpha}-n^{1-\alpha}} \\
& =\lim _{n \rightarrow \infty} \frac{1 /(n+1)}{1-(n /(n+1))^{1-\alpha}}=\lim _{n \rightarrow \infty} \frac{1 /(1+n)^{2}}{(1-\alpha)(n /(n+1))^{-\alpha} 1 /(n+1)^{2}} \\
& =\frac{1}{1-\alpha} .
\end{aligned}
$$

Remark 2.5. The Cesàro method $C^{s}$ for $s \in N$ fulfils equation (2.2).

Proof. $C^{s}$ is a matrix method generated by the Hausdorff moment sequence $\mu_{n}^{s}=\frac{s !}{(n+1)(n+2) \cdots(n+s)}$. Recall that

$$
\frac{s !}{(n+1)(n+2) \cdots(n+s)}=s \sum_{j=0}^{s-1} \frac{\left(\begin{array}{c}
s-1 \\
j
\end{array}\right)(-1)^{j}}{(n+1+j)} .
$$

We prove (2.2). Let $\lambda_{n k}^{s}$ be the Cesàro method $C^{s}$. Applying (2.9), we obtain

$$
\begin{aligned}
\lambda_{n k}^{s} & =\left(\begin{array}{c}
n \\
k
\end{array}\right) \Delta^{n-k} \mu_{k}^{s}=\left(\begin{array}{l}
n \\
k
\end{array}\right) \Delta^{n-k} s \sum_{j=0}^{s-1} \frac{\left(\begin{array}{c}
s-1 \\
j
\end{array}\right)(-1)^{j}}{k+1+j} \\
& =s \sum_{j=0}^{s-1}\left(\begin{array}{c}
s-1 \\
j
\end{array}\right)(-1)^{j}\left(\begin{array}{l}
n \\
k
\end{array}\right) \Delta^{n-k} \frac{1}{k+1+j} \\
& =s \sum_{j=0}^{s-1}\left(\begin{array}{c}
s-1 \\
j
\end{array}\right)(-1)^{j}\left(\begin{array}{l}
n \\
k
\end{array}\right) \Delta^{n-k} \mu_{k+j}^{1} .
\end{aligned}
$$

For $j=0$, the matrix $C_{j}=\left[c_{n k}^{j}\right]$, where $c_{n k}^{j}=\left(\begin{array}{l}n \\ k\end{array}\right) \Delta^{n-k} \mu_{k+j}^{1}$ is the Cesàro $C^{1}$ method that satisfies (2.2), which follows from Remark 2.4. For $j>0$, the 
method $C_{j}$ is the matrix generated on the basis of the right-shifting sequence of moments $\mu_{k+j}^{1}$. Therefore, $C_{j}$ satisfies the condition (2.2) for $j>0$, which follows from Corollary 2.3. Thus, $\sum_{k=0}^{n} \frac{\left|\lambda_{n k}^{s}\right|}{(k+1)^{\alpha}}=O\left(\frac{1}{(n+1)^{\alpha}}\right)$.

Remark 2.6. Let $A$ be a Hölder method $H^{s}$ for $s \in N$, where $H^{s}$ is the Hausdorff matrix, which is generated by the Hausdorff moment sequence $\mu_{n}^{H^{s}}=\frac{1}{(n+1)^{s}}$. Therefore, $A$ satisfies the condition (2.3) in Theorem 2.1.

Proof. Every Hausdorff matrix $\Lambda$ may be formulated as a product of matrices $\delta \mu \delta$, where $\delta_{n k}=(-1)^{k}\left(\begin{array}{l}n \\ k\end{array}\right)$ for $n \geq k \geq 0$ and 0 for $n<k$. The matrix $\mu$ is a diagonal matrix with elements of the main diagonal $\mu_{n}^{H^{s}}=\frac{1}{(n+1)^{s}}$ in the case of Hölder's $s$-th order method. Because $\delta^{-1}=\delta$, the Hausdorff matrix for the $H^{s}$ method may be formulated as follows:

$$
\Lambda^{H^{s}}=\delta \mu^{H^{s}} \delta=\delta \mu^{H^{1}} \cdots \mu^{H^{1}} \delta=\delta \mu^{H^{1}} \delta \cdots \delta \mu^{H^{1}} \delta=\Lambda^{H^{1}} \cdots \Lambda^{H^{1}} .
$$

Because Hölder's first order method is identical to Cesàro's first order method, and in view of Remark 2.4 and the inequalities in (2.5) and (2.6), we obtain (2.3).

In view of Theorem 2.1 and Remark 2.6, we obtain the following:

Theorem 2.7. Let $f$ be a $2 \pi$ periodic function and Lebesgue integrable on $[-\pi, \pi]$, which satisfies Lipschitz condition (2.1). If $0<\alpha<1$ and $r \in N$, then

$$
\left\|H^{r}\left(s_{n}\right)-\bar{f}\right\|_{\infty}=O\left(\frac{1}{(n+1)^{\alpha}}\right) .
$$

Based on Corollary 2.3, we conclude that the product of the Cesàro $C^{1}$ method and Hausdorff matrix $A$, generated on the basis of a right-shifting sequence of moments, fulfils the condition (2.3). As a result, a question arises: can the Cesàro $C^{1}$ method be extended to any of the Hausdorff $B$ matrices so that condition (2.3) is still fulfilled? Based on the case of $\alpha \in$ $(0,1 / 2]$, we prove that such a generalization is not possible. For the purpose of this proof, $A$ is the Euler method $(E, 1)$ and $B$ is the Hausdorff method generated by the function $\chi(x)=x^{\beta}$ for $0<\beta<\alpha$, which satisfies the assumptions in (1.3). The details are formulated in the following theorem:

Theorem 2.8. We define the function $f$, which is $2 \pi$ periodic, Lebesgue integrable on $[0,2 \pi]$ and satisfies the Lipschitz condition (2.1) for $\alpha \in(0,1 / 2]$ :

$$
f(x)=\left\{\begin{array}{ll}
\sin ^{\alpha} \frac{x-2 k \pi}{2} & \text { if } x \in[2 k \pi,(2 k+1) \pi) \\
-\sin \frac{x-2 k \pi}{2} & \text { if } x \in[(2 k-1) \pi, 2 k \pi)
\end{array} \quad k \in Z .\right.
$$

Let $s_{n}(x)$ represent the $n$-th partial sum of a conjugate series of the Fourier series of $f$ at a point $x$ and $\bar{f}$ be defined by (2.4). Then, there exists a class 
of Hausdorff methods $L=\{\Lambda\}$ such that

$$
\forall \Lambda \in L \lim _{n \rightarrow \infty}(n+1)^{\alpha} \operatorname{ess~sup}_{x \in[0,2 \pi]}\left|\Lambda\left(s_{n}(x)-\bar{f}(x)\right)\right|=+\infty .
$$

Note that Rhoades, Ozkoklu and Albayrak [10] proved the following theorem:

Theorem 2.9. Let $f$ be a $2 \pi$-periodic function and Lebesgue integrable on $[-\pi, \pi]$, and let it belong to the Lipschitz class Lip $\alpha$ for $0<\alpha<1$. Let $s_{n}(x)$ represent the $n$-th partial sum of series of the Fourier series of $f$ at a point $x$. Then,

$$
\exists M>0 \forall n \geq 0(n+1)^{\alpha} \sup _{x \in[0,2 \pi]}\left|\Lambda\left(s_{n}(x)-f(x)\right)\right|<M .
$$

We return to Theorem 2.8.

Proof. For the purpose of the following proof, $\alpha \in(0,1 / 2]$ is fixed. We define $\Lambda=\Lambda^{\beta} *(E, 1)$, where $(E, 1)$ is the Euler method and $\Lambda^{\beta}$ is the Hausdorff method generated by the function $\chi(x)=x^{\beta}$ and satisfying the assumption

$$
0<\beta<\alpha
$$

We know that the sequence

$$
z_{n}\left(x_{0}\right)=s_{n}\left(x_{0}\right)-\bar{f}\left(x_{0}\right)=\frac{1}{\pi} \int_{0}^{\pi} \Psi_{x_{0}}(t) \frac{\cos (n+1 / 2) t d t}{2 \sin (t / 2)} d t
$$

tends to zero with respect to $n$.

Note that

$$
\begin{aligned}
\sum_{k=0}^{n}\left(\begin{array}{l}
n \\
k
\end{array}\right) \frac{\cos (k+1 / 2) t}{2 \sin (t / 2)} \\
=\sum_{k=0}^{n}\left(\begin{array}{l}
n \\
k
\end{array}\right) \frac{(\cos t+i \sin t)^{k+1}-(\cos t-i \sin t)^{k+1}-(\cos t+i \sin t)^{k}+(\cos t-i \sin t)^{k}}{8 i \sin ^{2}(t / 2)} \\
=\frac{(\cos t-1+i \sin t)(\cos t+1+i \sin t)^{n}-(\cos t+1-i \sin t)^{n}(\cos t-1-i \sin t)}{8 i \sin ^{2}(t / 2)} \\
=\frac{2^{n} \cos ^{n}(t / 2)}{4 i \sin (t / 2)}\left[(-\sin (t / 2)+i \cos (t / 2))(\cos (t / 2)+i \sin (t / 2))^{n}\right. \\
\left.\quad+(\sin (t / 2)+i \cos (t / 2))(\cos (t / 2)-i \sin (t / 2))^{n}\right] \\
=\frac{2^{n-1} \cos ^{n}(t / 2) \cos \frac{(n+1) t}{2}}{\sin (t / 2)} .
\end{aligned}
$$


Denoting the Euler (E,1) matrix summability transform of $z_{n}\left(x_{0}\right)$ by $w_{n}\left(x_{0}\right)$, we obtain

$$
w_{n}\left(x_{0}\right)=\frac{1}{2^{n}} \sum_{k=0}^{n}\left(\begin{array}{l}
n \\
k
\end{array}\right) z_{k}\left(x_{0}\right)=\frac{1}{2 \pi} \int_{0}^{\pi} \Psi_{x_{0}}(t) \frac{\cos ^{n}(t / 2) \cos \frac{(n+1)}{2} t}{\sin (t / 2)} d t .
$$

Assume that

$$
x_{0} \in\left(0,\left(\frac{\alpha \pi}{8 s(1+\alpha)(n+1)^{2}}\right)^{\frac{1}{1-\alpha}}\right), s \geq 3, \alpha \in(0,1 / 2] .
$$

A direct calculation provides

$$
\Psi_{x_{0}}(t)= \begin{cases}\sin ^{\alpha} \frac{x_{0}+t}{2}-\sin ^{\alpha} \frac{x_{0}-t}{2} & \text { if } t \in\left[0, x_{0}\right] \\ \sin ^{\alpha} \frac{x_{0}+t}{2}+\sin \frac{x_{0}-t}{2} & \text { if } t \in\left(x_{0}, \pi-x_{0}\right) \\ -\sin \frac{x_{0}+t-2 \pi}{2}+\sin \frac{x_{0}-t}{2} & \text { if } t \in\left[\pi-x_{0}, \pi\right]\end{cases}
$$

for $t \in[0, \pi]$.

Let

$$
\begin{aligned}
& w_{n}^{1}\left(x_{0}\right)=\int_{0}^{x_{0}}\left(\sin ^{\alpha} \frac{x_{0}+t}{2}-\sin ^{\alpha} \frac{x_{0}-t}{2}\right) \frac{\cos ^{n}(t / 2) \cos \frac{n+1}{2} t}{\sin (t / 2)} d t, \\
& w_{n}^{2}\left(x_{0}\right)=\int_{x_{0}}^{\pi-x_{0}}\left(\sin ^{\alpha} \frac{x_{0}+t}{2}+\sin \frac{x_{0}-t}{2}\right) \frac{\cos ^{n}(t / 2) \cos \frac{n+1}{2} t}{\sin (t / 2)} d t, \\
& w_{n}^{3}\left(x_{0}\right)=\int_{\pi-x_{0}}^{\pi}\left(-\sin \frac{x_{0}+t-2 \pi}{2}+\sin \frac{x_{0}-t}{2}\right) \frac{\cos ^{n}(t / 2) \cos \frac{n+1}{2} t}{\sin (t / 2)} d t .
\end{aligned}
$$

It is clear that

$$
\begin{gathered}
w_{0}^{1}\left(x_{0}\right) \geq 0, \\
w_{n}^{3}\left(x_{0}\right) \geq 0 \text { for } n=0, n=4 r-1, n=4 r, r \in N .
\end{gathered}
$$

Note that if $0<y<x<1$ and $b=[1 / \alpha]$, then

$$
\begin{aligned}
& (x-y)\left(x^{1 / \alpha-1}+y x^{1 / \alpha-2}+y^{2} x^{1 / \alpha-3}+\ldots+y^{b-1} x^{1 / \alpha-b}+y^{b} x^{1 / \alpha-(b+1)}\right) \\
& =\left(x^{1 / \alpha}-y^{1 / \alpha}\right)-\left(y^{b+1} x^{1 / \alpha-(b+1)}-y^{1 / \alpha}\right)
\end{aligned}
$$

where $y^{b+1} x^{1 / \alpha-(b+1)}-y^{1 / \alpha} \leq 0$. 
Let $x=\sin ^{\alpha}\left(\frac{x_{0}+t}{2}\right)$ and $y=\sin ^{\alpha}\left(\frac{x_{0}-t}{2}\right)$ for $t \in\left(0, x_{0}\right), n \geq 1, s \geq 3$. Then

$$
\begin{aligned}
\frac{\sin ^{\alpha}\left(\frac{x_{0}+t}{2}\right)-\sin ^{\alpha}\left(\frac{x_{0}-t}{2}\right)}{\sin (t / 2)} & \geq \frac{\sin \left(\frac{x_{0}+t}{2}\right)-\sin \left(\frac{x_{0}-t}{2}\right)}{\sin (t / 2) \sum_{j=1}^{b+1}\left(\sin ^{\alpha}\left(\frac{x_{0}+t}{2}\right)\right)^{1 / \alpha-j}\left(\sin ^{\alpha}\left(\frac{x_{0}-t}{2}\right)\right)^{j-1}} \\
& =\frac{2 \cos \left(x_{0} / 2\right)}{\sum_{j=1}^{b+1}\left(\sin ^{\alpha}\left(\frac{x_{0}+t}{2}\right)\right)^{1 / \alpha-j}\left(\sin ^{\alpha}\left(\frac{x_{0}-t}{2}\right)\right)^{j-1}} \\
& \geq \frac{2 \cos \left(x_{0} / 2\right)}{(b+1) \sin ^{1-\alpha}\left(\frac{x_{0}+t}{2}\right)} \geq \frac{2 \cos \left(x_{0} / 2\right)}{(1+1 / \alpha) \frac{\alpha \pi}{8 s(1+\alpha)(n+1)^{2}}} \\
& \geq \frac{192 \cos \left(x_{0} / 2\right)}{\pi}
\end{aligned}
$$

This follows from (2.11).

Therefore,

$$
\begin{aligned}
\forall n \geq 1 w_{n}^{1}\left(x_{0}\right) & \geq \frac{192}{\pi} \cos ^{n+1}\left(x_{0} / 2\right) \int_{0}^{x_{0}} \cos \left(\frac{n+1}{2} t\right) d t \\
& =\frac{384}{\pi(n+1)} \cos ^{n+1}\left(x_{0} / 2\right) \sin \left(\frac{n+1}{2} x_{0}\right) \\
& \geq \frac{384}{\pi(n+1)} \cos ^{n+1}\left(x_{0} / 2\right) \sin \left(\frac{n+1}{4} x_{0}\right) .
\end{aligned}
$$

If $n=4 r-1$ or $n=4 r-2$, then

$$
\begin{aligned}
& w_{n}^{3}\left(x_{0}\right)=\int_{\pi-x_{0}}^{\pi} \frac{2 \cos (t / 2) \sin \left(x_{0} / 2\right)}{\sin (t / 2)} \cos ^{n}(t / 2) \cos \left(\frac{n+1}{2} t\right) d t \\
& \geq \frac{2 \cos \frac{\pi-x_{0}}{2} \sin \left(x_{0} / 2\right)}{\sin \frac{\pi-x_{0}}{2}} \cos ^{n} \frac{\pi-x_{0}}{2} \frac{2}{n+1}\left[\sin \left(\frac{n+1}{2} \pi\right)\right. \\
& \left.-\sin \frac{(n+1)\left(\pi-x_{0}\right)}{2}\right] \geq-\frac{16}{n+1} \cos ^{n+1}\left(x_{0} / 2\right) \sin \frac{(n+1) x_{0}}{4} .
\end{aligned}
$$

Therefore,

$$
\forall n \geq 0 w_{n}^{1}\left(x_{0}\right)+w_{n}^{3}\left(x_{0}\right) \geq 0
$$

This follows from (2.11), (2.12), (2.13), (2.14) and (2.15).

A direct calculation provides

$$
\exists q^{\prime}>0 w_{n}^{2}\left(x_{0}\right)>q^{\prime} \text { for } 0 \leq n \leq 3 .
$$


If $n=1$, then

$$
\begin{aligned}
w_{1}^{2}\left(x_{0}\right) & =\int_{x_{0}}^{\pi-x_{0}} \frac{\sin ^{\alpha}\left(\frac{x_{0}}{2}+\frac{t}{2}\right)+\sin \left(\frac{x_{0}}{2}-\frac{t}{2}\right)}{\sin \frac{t}{2}} \cos \frac{t}{2} \cos t d t \\
& =\int_{x_{0}}^{\pi / 2} \frac{\sin ^{\alpha}\left(\frac{x_{0}}{2}+\frac{t}{2}\right)+\sin \left(\frac{x_{0}}{2}-\frac{t}{2}\right)}{\sin \frac{t}{2}} \cos \frac{t}{2} \cos t d t \\
& +\int_{\pi / 2}^{\pi-x_{0}} \frac{\sin ^{\alpha}\left(\frac{x_{0}}{2}+\frac{t}{2}\right)+\sin \left(\frac{x_{0}}{2}-\frac{t}{2}\right)}{\sin \frac{t}{2}} \cos \frac{t}{2} \cos t d t=C_{1}+C_{2},
\end{aligned}
$$

where

$C_{1} \geq \int_{x_{0}}^{\pi / 2} \frac{\cos \frac{t}{2}\left(2 \cos ^{2} \frac{t}{2}-1\right)}{\sin ^{1 / 2}\left(\frac{t}{2}\right)} d t-\int_{0}^{\pi / 2} \cos \frac{t}{2}\left(1-2 \sin ^{2} \frac{t}{2}\right) d t>2.69-0.32$

and

$$
C_{2} \geq \sqrt{2} \int_{\pi / 2}^{\pi} \cos \frac{t}{2}\left(1-2 \sin ^{2} \frac{t}{2}\right) d t>-0.4 .
$$

If $n=2$, then

$$
\begin{aligned}
w_{2}^{2}\left(x_{0}\right) & =\int_{x_{0}}^{\pi-x_{0}} \frac{\sin ^{\alpha}\left(\frac{x_{0}}{2}+\frac{t}{2}\right)+\sin \left(\frac{x_{0}}{2}-\frac{t}{2}\right)}{\sin \frac{t}{2}} \cos ^{2} \frac{t}{2} \cos \frac{3 t}{2} d t \\
& =\int_{x_{0}}^{\pi / 3} \frac{\sin ^{\alpha}\left(\frac{x_{0}}{2}+\frac{t}{2}\right)+\sin \left(\frac{x_{0}}{2}-\frac{t}{2}\right)}{\sin \frac{t}{2}} \cos ^{2} \frac{t}{2} \cos \frac{3 t}{2} d t \\
& +\int_{\pi / 3}^{\pi-x_{0}} \frac{\sin ^{\alpha}\left(\frac{x_{0}}{2}+\frac{t}{2}\right)+\sin \left(\frac{x_{0}}{2}-\frac{t}{2}\right)}{\sin \frac{t}{2}} \cos ^{2} \frac{t}{2} \cos \frac{3 t}{2} d t=C_{1}+C_{2},
\end{aligned}
$$

where

$$
C_{1} \geq \int_{x_{0}}^{\pi / 3} \frac{1}{\sin ^{1-\alpha} \frac{t}{2}} \cos ^{2} \frac{t}{2} \cos \frac{3 t}{2} d t-\int_{x_{0}}^{\pi / 3} \cos ^{2} \frac{t}{2} \cos \frac{3 t}{2} d t>2-0.7
$$

and

If $n=3$, then

$$
C_{2} \geq 2 \int_{\pi / 3}^{\pi} \cos ^{2} \frac{t}{2} \cos \frac{3 t}{2}>-0.8
$$

$$
\begin{aligned}
w_{3}^{2}\left(x_{0}\right) & =\int_{x_{0}}^{\pi-x_{0}} \frac{\sin ^{\alpha}\left(\frac{x_{0}}{2}+\frac{t}{2}\right)+\sin \left(\frac{x_{0}}{2}-\frac{t}{2}\right)}{\sin \frac{t}{2}} \cos ^{3} \frac{t}{2} \cos 2 t d t \\
& =\int_{x_{0}}^{\pi / 4} \frac{\sin ^{\alpha}\left(\frac{x_{0}}{2}+\frac{t}{2}\right)+\sin \left(\frac{x_{0}}{2}-\frac{t}{2}\right)}{\sin \frac{t}{2}} \cos ^{3} \frac{t}{2} \cos 2 t d t \\
& +\int_{\pi / 4}^{3 \pi / 4} \frac{\sin ^{\alpha}\left(\frac{x_{0}}{2}+\frac{t}{2}\right)+\sin \left(\frac{x_{0}}{2}-\frac{t}{2}\right)}{\sin \frac{t}{2}} \cos ^{3} \frac{t}{2} \cos 2 t d t \\
& +\int_{3 \pi / 4}^{\pi-x_{0}} \frac{\sin ^{\alpha}\left(\frac{x_{0}}{2}+\frac{t}{2}\right)+\sin \left(\frac{x_{0}}{2}-\frac{t}{2}\right)}{\sin \frac{t}{2}} \cos ^{3} \frac{t}{2} \cos 2 t d t=C_{1}+C_{2}+C_{3},
\end{aligned}
$$


where

$$
\begin{aligned}
C_{1} & \geq \int_{x_{0}}^{\pi / 4} \frac{1}{\sin ^{1 / 2}\left(\frac{t}{2}\right)} \cos ^{3} \frac{t}{2} \cos 2 t d t-\int_{x_{0}}^{\pi / 4} \cos ^{3} \frac{t}{2} \cos 2 t d t>1.9-0.5, \\
C_{2} & \geq \int_{\pi / 4}^{3 \pi / 4} \frac{\sin ^{\alpha}\left(\frac{x_{0}}{2}+\frac{t}{2}\right)}{\sin \frac{t}{2}} \cos ^{3} \frac{t}{2} \cos 2 t d t \geq \int_{\pi / 4}^{3 \pi / 4} \frac{\cos ^{3} \frac{t}{2} \cos 2 t}{\sin \frac{\pi}{8}} d t>-0.97
\end{aligned}
$$

and $C_{3}>0$.

Now we consider $n \geq 4$.

We define

$$
I_{m}^{n}=\left[\int_{\frac{\pi}{(m+1)(n+1)}}^{\frac{\pi}{m(n+1)}}+\int_{\frac{\pi}{(n+1)}\left(1+\frac{m-1}{m}\right)}^{\frac{\pi}{(n+1)}\left(1+\frac{m}{m+1}\right)}\right] \frac{\sin ^{\alpha} \frac{t+x_{0}}{2}}{\sin (t / 2)} \cos ^{n}(t / 2) \cos \frac{(n+1) t}{2} d t
$$

where $1 \leq m \leq s-1$,

$$
\begin{gathered}
I_{s}^{n}=\left[\int_{x_{0}}^{\frac{\pi}{s(n+1)}}+\int_{\frac{\pi}{(n+1)}\left(1+\frac{s-1}{s}\right)}^{\frac{3 \pi}{(n+1)}}\right] \frac{\sin ^{\alpha} \frac{t+x_{0}}{2}}{\sin (t / 2)} \cos ^{n}(t / 2) \cos \frac{(n+1) t}{2} d t \\
J_{l}^{n}=\left[\int_{\frac{\pi(p+l)}{2 p(n+1)}}^{\frac{\pi(p+l+1)}{2 p(n+1)}}+\int_{\frac{\pi(3 p-l-1)}{2 p(n+1)}}^{\frac{\pi(3 p-l)}{2 p(n+1)}}\right] \frac{\sin ^{\alpha} \frac{t+x_{0}}{2}}{\sin (t / 2)} \cos ^{n}(t / 2) \cos \frac{(n+1) t}{2} d t
\end{gathered}
$$

for $p \geq 2$ and $0 \leq l \leq p-2$.

Note that

$$
\begin{aligned}
I_{m}^{n} \geq & \frac{2}{n+1}\left(\cos \frac{\pi(2 m-1)}{2 m(n+1)}\right)^{n} \frac{\sin ^{\alpha}\left(\frac{x_{0}}{2}+\frac{\pi(2 m-1)}{2 m(n+1)}\right)}{\sin \frac{\pi(2 m-1)}{2 m(n+1)}} \\
& \times\left[\frac{\sin \frac{\pi(2 m-1)}{2 m(n+1)}}{\sin \frac{\pi}{2 m(n+1)}} \frac{\sin ^{\alpha}\left(\frac{x_{0}}{2}+\frac{\pi}{2 m(n+1)}\right)}{\sin ^{\alpha}\left(\frac{x_{0}}{2}+\frac{\pi(2 m-1)}{2 m(n+1)}\right)}-1\right]\left(\sin \frac{\pi}{2 m}-\sin \frac{\pi}{2(m+1)}\right) .
\end{aligned}
$$

This follows from (2.18).

We can prove that

$$
\frac{\sin \left(\frac{x_{0}}{2}+\frac{\pi}{2 m(n+1)}\right)}{\sin \left(\frac{x_{0}}{2}+\frac{\pi(2 m-1)}{2 m(n+1)}\right)}
$$

is non-increasing with respect to $x_{0} \in(0, \pi / 2)$.

After considering $\cot \frac{\pi}{2 m(n+1)} \geq(2 m-1) \cot \frac{\pi(2 m-1)}{2 m(n+1)}$, we can prove that

$$
\frac{\sin \frac{\pi(2 m-1)}{2 m(n+1)}}{\sin \frac{\pi}{2 m(n+1)}}
$$

is non-decreasing with respect to $n$ for $m \in\{1, \ldots, s\}$. 
Furthermore,

$$
\frac{\sin ^{\alpha}\left(\frac{x_{0}}{2}+\frac{\pi(2 m-1)}{2 m(n+1)}\right)}{\sin \frac{\pi(2 m-1)}{2 m(n+1)}}
$$

is non-increasing with respect to $m$. In view of (2.21), (2.22) and (2.23), the following inequality is satisfied:

$$
\begin{aligned}
& I_{m}^{n} \geq\left(\cos \frac{\pi(2 s-1)}{2 s(n+1)}\right)^{n} \frac{\sin ^{\alpha}\left(\frac{x_{0}}{2}+\frac{\pi(2 s-1)}{2 s(n+1)}\right)}{\sin \frac{\pi(2 s-1)}{2 s(n+1)}} \\
& \times\left[\left(\frac{\sin \frac{\pi(2 m-1)}{10 m}}{\sin \frac{\pi}{10 m}}\right)^{1-\alpha}-1\right] \frac{2}{n+1}\left(\sin \frac{\pi}{2 m}-\sin \frac{\pi}{2(m+1)}\right) .
\end{aligned}
$$

We define

$$
H_{m}^{\alpha}=\left[\left(\frac{\sin \frac{\pi(2 m-1)}{10 m}}{\sin \frac{\pi}{10 m}}\right)^{1-\alpha}-1\right]\left(\sin \frac{\pi}{2 m}-\sin \frac{\pi}{2(m+1)}\right) .
$$

We estimate

$$
\begin{aligned}
I_{s}^{n} \geq & \frac{2}{n+1}\left(\cos \frac{\pi(2 s-1)}{2 s(n+1)}\right)^{n} \frac{\sin ^{\alpha}\left(\frac{x_{0}}{2}+\frac{\pi(2 s-1)}{2 s(n+1)}\right)}{\sin \frac{\pi(2 s-1)}{2 s(n+1)}} \\
& \times\left[\left(\frac{\sin \frac{\pi(2 s-1)}{10 s}}{\sin \frac{\pi}{10 s}}\right)^{1-\alpha}\left(\sin \frac{\pi}{2 s}-\sin \frac{\pi}{40 s}\right)-\left(1+\sin \frac{\pi}{2 s}\right)\right],
\end{aligned}
$$

which follows from (2.11), (2.19), (2.21) and (2.22).

The mapping (2.20) can be estimated in similar ways, namely

$$
\begin{aligned}
J_{l}^{n} \geq & \cos ^{n} \frac{\pi(3 p-l-1)}{4 p(n+1)}\left[\sin \frac{\pi(p+l+1)}{4 p}-\sin \frac{\pi(p+l)}{4 p}\right] \\
& \times \frac{2}{(n+1)\left(\sin \frac{\pi(3 p-l-1)}{4(n+1) p}\right)^{1-\alpha}}\left[\left(\frac{\sin \frac{\pi(p+l+1)}{4 p(n+1)}}{\sin \frac{\pi(3 p-l-1)}{4 p(n+1)}}\right)^{\alpha-1}-1\right]
\end{aligned}
$$

for $p \geq 2$ and $l=0,1, \ldots, p-2$.

Note that

(2.28) $\quad \cos ^{n} \frac{\pi(3 p-l-1)}{4 p(n+1)}$ is increasing with respect to $n$. 
In view of (2.27) and (2.28), we obtain

$$
\begin{aligned}
J_{l}^{n} \geq & \frac{2}{n+1}\left[\sin \frac{\pi(p+l+1)}{4 p}-\sin \frac{\pi(p+l)}{4 p}\right] \\
& \times \frac{\left(\cos \frac{\pi(3 p-l-1)}{20 p}\right)^{4}}{\left(\sin \frac{\pi(3 p-l-1)}{20 p}\right)^{1-\alpha}}\left[\left(\frac{\sin \frac{\pi(3 p-l-1)}{20 p}}{\sin \frac{\pi(p+l+1)}{20 p}}\right)^{1-\alpha}-1\right]
\end{aligned}
$$

for $l=0,1, \ldots, p-2$.

We fix $p=5$ and $s=18$. We investigate the inequality (2.26) and let

$$
\begin{aligned}
\hat{I}_{18}^{n} & =\frac{2}{n+1}\left(\cos \frac{\pi(2 \cdot 18-1)}{2 \cdot 18(n+1)}\right)^{n} \frac{\sin ^{\alpha}\left(\frac{x_{0}}{2}+\frac{\pi(2 \cdot 18-1)}{2 \cdot 18(n+1)}\right)}{\sin \frac{\pi(2 \cdot 18-1)}{2 \cdot 18(n+1)}} \\
& \times\left[\left(\frac{\sin \frac{\pi(2 \cdot 18-1)}{10 \cdot 18}}{\sin \frac{\pi}{10 \cdot 18}}\right)^{1-\alpha}\left(\sin \frac{\pi}{2 \cdot 18}-\sin \frac{\pi}{40 \cdot 18}\right)-\sin \frac{\pi}{2 \cdot 18}\right] .
\end{aligned}
$$

Then

$$
\frac{\sin \frac{\pi}{2 \cdot 18}-\sin \frac{\pi}{40 \cdot 18}}{\sin \frac{\pi}{2 \cdot 18}}>0.949
$$

After considering that $\cos ^{n} \frac{\pi(2 s-1)}{2 s(n+1)}$ is increasing with respect to $n$, we obtain

$$
\hat{I}_{18}^{n} \geq \frac{2}{n+1} \frac{0.45}{\sqrt{0.573}}[\sqrt{32.85} \cdot 0.0828-0.0873]>\frac{2}{n+1} \cdot 0.23 .
$$

This follows from (2.22), (2.30) and (2.31).

The remaining part of the inequality (2.26) shall be denoted as

$$
\check{I}_{18}^{n}=-\frac{2}{n+1}\left(\cos \frac{\pi(2 \cdot 18-1)}{2 \cdot 18(n+1)}\right)^{n} \frac{\left(\sin \left(\frac{x_{0}}{2}+\frac{\pi(2 \cdot 18-1)}{2 \cdot 18(n+1)}\right)\right)^{\alpha}}{\sin \frac{\pi(2 \cdot 18-1)}{2 \cdot 18(n+1)}}
$$

In view of inequality (2.29), we obtain

$$
\begin{aligned}
\sum_{l=0}^{3} J_{l}^{n} \geq & \sum_{l=0}^{3} \frac{2}{n+1}\left[\sin \frac{\pi(6+l)}{20}-\sin \frac{\pi(5+l)}{20}\right] \frac{\left(\cos \frac{\pi(14-l)}{100}\right)^{4}}{\sqrt{\sin \frac{\pi(14-l)}{100}}} \\
& \times\left[\sqrt{\frac{\sin \frac{\pi(14-l)}{100}}{\sin \frac{\pi(6+l)}{100}}}-1\right]>\frac{2}{n+1} 0.1064 .
\end{aligned}
$$

Hence,

$$
\hat{I}_{18}^{n}+\sum_{l=0}^{3} J_{l}^{n}>\frac{2}{n+1} 0.3364
$$


This is true as a result of (2.32).

We can calculate that

$$
\sum_{m=2}^{17} H_{m}^{\alpha}>1.02 \text { for } \alpha \leq \frac{1}{2} .
$$

We estimate

$$
\sum_{m=2}^{17} I_{m}^{n} \geq \frac{2}{n+1} \cos ^{n} \frac{\pi(2 \cdot 18-1)}{2 \cdot 18(n+1)} \frac{\left(\sin \left(\frac{x_{0}}{2}+\frac{\pi(2 \cdot 18-1)}{2 \cdot 18(n+1)}\right)\right)^{\alpha}}{\sin \frac{\pi(2 \cdot 18-1)}{2 \cdot 18(n+1)}} 1.02 .
$$

This follows from (2.24), (2.25) and (2.36). After considering that the mapping

$$
\frac{\sin ^{\alpha} \frac{x_{0}+t}{2}}{\sin (t / 2)} \cos ^{n}(t / 2)
$$

is positive and increasing with respect to $t \in\left[\frac{\pi(2 p-1)}{2 p(n+1)}, \frac{\pi(2 p+1)}{2 p(n+1)}\right]$, we can estimate

$$
\begin{aligned}
\int_{x_{0}}^{\frac{3 \pi}{n+1}} & \frac{\sin ^{\alpha} \frac{t+x_{0}}{2}}{\sin (t / 2)} \cos ^{n}(t / 2) \cos \frac{(n+1) t}{2} d t=\int_{x_{0}}^{\frac{\pi}{s(n+1)}}+\int_{\frac{\pi(2 s-1)}{(n+1) s}}^{\frac{3 \pi}{n+1}} \\
& +\sum_{m=2}^{s-1}\left(\int_{\frac{\pi}{(m+1)(n+1)}}^{\frac{\pi}{m(n+1)}}+\int_{\frac{\pi(2 m-1)}{m(n+1)}}^{\frac{\pi(2 m+1)}{(m+1)(n+1)}}\right)+\sum_{l=0}^{p-2}\left(\int_{\frac{\pi(p+l)}{2 p(n+1)}}^{\frac{\pi(p+l+1)}{2 p(n+1)}}+\int_{\frac{\pi(3 p-l-1)}{2 p(n+1)}}^{\frac{\pi(3 p-l)}{2 p(n+1)}}\right) \\
& +\left(\int_{\frac{\pi(2 p-1)}{2 p(n+1)}}^{\frac{\pi}{n+1}}+\int_{\frac{\pi}{n+1}}^{\frac{\pi(2 p+1)}{2 p(n+1)}}\right) \geq I_{s}^{n}+\sum_{m=2}^{s-1} I_{m}^{n}+\sum_{l=0}^{p-2} J_{l}^{n} .
\end{aligned}
$$

Therefore,

$$
\begin{aligned}
& \forall n \geq 4 \forall \alpha \in(0,1 / 2] \forall x_{0} \in\left(0,\left(\frac{\pi \alpha}{8 \cdot 18(1+\alpha)(n+1)^{2}}\right)^{\frac{1}{1-\alpha}}\right) \\
& \int_{x_{0}}^{\frac{3 \pi}{n+1}} \frac{\sin ^{\alpha} \frac{t+x_{0}}{2}}{\sin (t / 2)} \cos ^{n}(t / 2) \cos \frac{(n+1) t}{2} d t \\
& \quad \geq \frac{2}{n+1}\left[0.3364+0.02 \cos ^{n} \frac{\pi(2 \cdot 18-1)}{2 \cdot 18(n+1)} \sin ^{\alpha-1} \frac{\pi(2 \cdot 18-1)}{2 \cdot 18(n+1)}\right]
\end{aligned}
$$

for $p=5$ and $s=18$. This follows from (2.33), (2.35) and (2.37).

We estimate the following integral for $n \geq 4$ :

$$
\begin{aligned}
& \int_{x_{0}}^{\frac{3 \pi}{n+1}} \frac{\sin \frac{x_{0}-t}{2}}{\sin (t / 2)} \cos ^{n}(t / 2) \cos \frac{(n+1) t}{2} d t \\
& \quad \geq-\frac{2}{n+1}+\int_{\frac{\pi}{n+1}}^{\frac{3 \pi}{n+1}} \frac{\sin \frac{x_{0}-t}{2}}{\sin (t / 2)} \cos ^{n}(t / 2) \cos \frac{(n+1) t}{2} d t .
\end{aligned}
$$


After considering that the sequence $\frac{\sin \left(\frac{\pi}{a(n+1)^{2}}-\frac{b \pi}{n+1}\right)}{\sin \frac{b \pi}{n+1}}$ is decreasing with respect to $n$ for $a \in N$ and $b \in\left[\frac{1}{2}, \frac{4}{3}\right]$, we divide the interval $\left[\frac{\pi}{n+1}, \frac{3 \pi}{n+1}\right]$ so that

$$
\int_{\frac{\pi}{n+1}}^{\frac{3 \pi}{n+1}} \frac{\sin \frac{x_{0}-t}{2}}{\sin (t / 2)} \cos ^{n}(t / 2) \cos \frac{(n+1) t}{2} d t \geq \frac{2}{n+1} 0.752 .
$$

In view of (2.39) and (2.40), the following condition is satisfied:

$$
\begin{aligned}
& \forall n \geq 4 \forall \alpha \in(0,1 / 2] \forall x_{0} \in\left(0,\left(\frac{\pi \alpha}{8 \cdot 18(1+\alpha)(n+1)^{2}}\right)^{\frac{1}{1-\alpha}}\right) \\
& \int_{x_{0}}^{\frac{3 \pi}{n+1}} \frac{\sin \frac{x_{0}-t}{2}}{\sin (t / 2)} \cos ^{n}(t / 2) \cos \frac{(n+1) t}{2} d t \geq-0.248 \frac{2}{n+1} .
\end{aligned}
$$

Note that

$$
\begin{aligned}
& \forall n \geq 4 \forall \alpha \in(0,1 / 2] \forall x_{0} \in\left(0,\left(\frac{\pi \alpha}{8 \cdot 18(1+\alpha)(n+1)^{2}}\right)^{\frac{1}{1-\alpha}}\right) \\
& \int_{x_{0}}^{\frac{3 \pi}{n+1}} \frac{\sin ^{\alpha} \frac{t+x_{0}}{2}+\sin \frac{x_{0}-t}{2}}{\sin (t / 2)} \cos ^{n}(t / 2) \cos \frac{(n+1) t}{2} d t \\
& \quad \geq \frac{0.04}{n+1} \cos ^{n} \frac{\pi(2 \cdot 18-1)}{2 \cdot 18(n+1)} \sin ^{\alpha-1} \frac{\pi(2 \cdot 18-1)}{2 \cdot 18(n+1)}
\end{aligned}
$$

which follows from (2.38) and (2.41).

If $n \geq 4$, then the function

$$
\frac{\sin ^{\alpha} \frac{x_{0}+t}{2}+\sin \frac{x_{0}-t}{2}}{\sin (t / 2)}
$$

is positive and decreasing with respect to $t \in\left[\frac{3 \pi}{n+1}, \pi-x_{0}\right]$. Therefore,

$$
\int_{\frac{3 \pi}{n+1}}^{\pi-x_{0}} \frac{\sin ^{\alpha} \frac{x_{0}+t}{2}+\sin \frac{x_{0}-t}{2}}{\sin (t / 2)} \cos ^{n}(t / 2) \cos \frac{(n+1) t}{2} d t \geq 0 .
$$

In view of (2.42) and (2.43), we obtain

$$
\begin{aligned}
& \forall n \geq 4 \forall \alpha \in(0,1 / 2] \forall x_{0} \in\left(0,\left(\frac{\alpha \pi}{8 \cdot 18(1+\alpha)(n+1)^{2}}\right)^{\frac{1}{1-\alpha}}\right) \\
& \int_{x_{0}}^{\pi-x_{0}} \frac{\sin ^{\alpha} \frac{x_{0}+t}{2}+\sin \frac{x_{0}-t}{2}}{\sin (t / 2)} \cos ^{n}(t / 2) \cos \frac{(n+1) t}{2} d t \\
& \quad \geq \frac{0.04}{n+1} \cos ^{n} \frac{\pi(2 \cdot 18-1)}{2 \cdot 18(n+1)} \sin ^{\alpha-1} \frac{\pi(2 \cdot 18-1)}{2 \cdot 18(n+1)} .
\end{aligned}
$$


In view of $(2.16),(2.17)$ and $(2.44)$, we obtain

$$
\begin{aligned}
& \exists q>0 \forall n \geq 0 \forall \alpha \in(0,1 / 2] \forall x_{0} \in\left(0,\left(\frac{\alpha \pi \frac{1}{1+\alpha}}{8 \cdot 18(n+1)^{2}}\right)^{\frac{1}{1-\alpha}}\right) \\
& (n+1) w_{n}\left(x_{0}\right)>q .
\end{aligned}
$$

Let $\Lambda^{\beta}$ be a Hausdorff matrix with elements $\lambda_{m n}^{\beta}=\left(\begin{array}{c}m \\ n\end{array}\right) \Delta^{m-n} \mu_{n}^{\beta}$, where $\mu_{n}^{\beta}=\int_{0}^{1} x^{n} d x^{\beta}$ for $\beta \in(0, \alpha)$. Because the function $x^{\beta}$ increases with respect to $x \in[0,1]$, the following inequality is fulfilled:

$$
\Delta^{m-n} \mu_{n}^{\beta}=\int_{0}^{1} x^{n}(1-x)^{m-n} d x^{\beta} \geq 0 .
$$

Denoting the $\Lambda^{\beta}$ matrix transform of $w_{n}\left(x_{0}\right)$ by $v_{m}^{\beta}\left(x_{0}\right)$, we obtain

$$
v_{m}^{\beta}\left(x_{0}\right)=\sum_{n=0}^{m} \lambda_{m n}^{\beta} w_{n}\left(x_{0}\right), \text { where } x_{0} \in\left(0,\left(\frac{\alpha \pi \frac{1}{1+\alpha}}{8 \cdot 18(m+1)^{2}}\right)^{\frac{1}{1-\alpha}}\right) .
$$

We fix a real number $\mu_{-1}^{\beta}$. Then

$$
\begin{aligned}
v_{m}^{\beta}\left(x_{0}\right) & =\frac{1}{m+1} \sum_{n=0}^{m}\left(\begin{array}{c}
m+1 \\
n+1
\end{array}\right) \Delta^{m-n} \mu_{n}^{\beta}(n+1) w_{n}\left(x_{0}\right) \\
& \geq \frac{q}{m+1} \sum_{n=0}^{m}\left(\begin{array}{c}
m+1 \\
n+1
\end{array}\right) \Delta^{m-n} \mu_{n}^{\beta}
\end{aligned}
$$

for $x_{0} \in\left(0,\left(\frac{\alpha \pi \frac{1}{1+\alpha}}{8 \cdot 18(m+1)^{2}}\right)^{\frac{1}{1-\alpha}}\right)$, this follows from (2.45) and (2.46). Therefore,

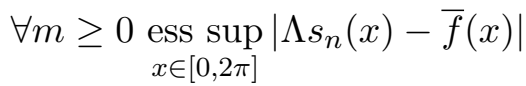

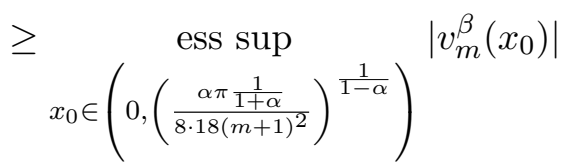

$$
\begin{aligned}
& \geq \frac{q}{m+1} \sum_{n=0}^{m}\left(\begin{array}{c}
m+1 \\
n+1
\end{array}\right) \Delta^{m-n} \mu_{n}^{\beta} \text {. }
\end{aligned}
$$


Note that for $m \geq 0$, the following condition is fulfilled:

$$
\begin{aligned}
& \frac{q}{m+1} \sum_{n=0}^{m}\left(\begin{array}{c}
m+1 \\
n+1
\end{array}\right) \Delta^{m-n} \mu_{n}^{\beta} \\
& =\frac{q}{m+1}\left(-\Delta^{m+1} \mu_{-1}^{\beta}+\Delta^{m+1} \mu_{-1}^{\beta}+\sum_{n=0}^{m}\left(\begin{array}{c}
m+1 \\
n+1
\end{array}\right) \Delta^{m-n} \mu_{n}^{\beta}\right) \\
& =\frac{q}{m+1}\left(-\Delta^{m+1} \mu_{-1}^{\beta}+\mu_{-1}^{\beta}\right) .
\end{aligned}
$$

For the purpose of the following proof we show that

$$
\forall \alpha \in\left(0, \frac{1}{2}\right] \frac{1}{(m+2)^{1-\alpha}-(m+1)^{1-\alpha}} \geq(m+2)^{\alpha},
$$

and

$$
-\Delta^{m+2} \mu_{-1}^{\beta}-\left(-\Delta^{m+1} \mu_{-1}^{\beta}\right)=\Delta^{m+1} \mu_{0}^{\beta} \geq 0 .
$$

Note that

$$
\Delta^{m+1} \mu_{0}^{\beta}=\prod_{j=1}^{m+1} \frac{j}{\beta+j} .
$$

This follows from (2.46). We estimate

$$
(m+1)^{\alpha} \operatorname{ess~sup}_{x \in[0,2 \pi]}\left|v_{m}^{\beta}(x)\right| \geq \frac{q(m+1)^{\alpha}}{m+1}\left(-\Delta^{m+1} \mu_{-1}^{\beta}+\mu_{-1}^{\beta}\right) .
$$

This follows from (2.48).

We calculate

$$
\begin{aligned}
& \lim _{m \rightarrow \infty} \frac{-\Delta^{m+1} \mu_{-1}^{\beta}}{(m+1)^{1-\alpha}}=\lim _{m \rightarrow \infty} \frac{-\Delta^{m+2} \mu_{-1}^{\beta}+\Delta^{m+1} \mu_{-1}^{\beta}}{(m+2)^{1-\alpha}-(m+1)^{1-\alpha}} \\
& \geq \lim _{m \rightarrow \infty} \Delta^{m+1} \mu_{0}^{\beta}(m+2)^{\alpha}=\lim _{m \rightarrow \infty} e^{\ln \left[(m+2)^{\alpha} \Delta^{m+1} \mu_{0}^{\beta}\right]} \\
& =\lim _{m \rightarrow \infty} e^{\left[\ln (m+2)^{\alpha}+\ln \prod_{j=1}^{m+1} \frac{j / \beta}{1+j / \beta}\right]} \\
& =\lim _{m \rightarrow \infty} \exp \left[\ln (m+2)^{\alpha}\left(\frac{\sum_{j=1}^{m+1} \ln \frac{j / \beta}{1+j / \beta}}{\ln (m+2)^{\alpha}}+1\right)\right] .
\end{aligned}
$$

This is a consequence of (2.49), (2.50) and (2.51).

Furthermore,

$$
\lim _{m \rightarrow \infty} \frac{\sum_{j=1}^{m+1} \ln \frac{j / \beta}{1+j / \beta}}{\ln (m+2)^{\alpha}}=\lim _{m \rightarrow \infty} \frac{\ln \frac{(m+2) / \beta}{1+(m+2) / \beta}}{\ln \left(\frac{m+3}{m+2}\right)^{\alpha}}=-\frac{\beta}{\alpha}>-1 .
$$


This follows from (2.10). In view of (2.53) and (2.54), we obtain

$$
\lim _{m \rightarrow \infty} \frac{-1}{(m+1)^{1-\alpha}} \Delta^{m+1} \mu_{-1}^{\beta}=+\infty \text {. }
$$

In view of (2.47), (2.52) and (2.55), our proof is complete.

\section{REFERENCES}

[1] Hardy, G. H., Divergent Series, Clarendon Press, Oxford, 1949.

[2] Hausdorff, F., Summationsmethoden und Momentfolgen, Math. Z. 9 (1921), I: 74109, II: $280-289$.

[3] Hildebrandt, T. H., Schoenberg, I. J., On linear functional operations and the moment problem for a finite interval in one or several dimensions, Ann. of Math. 34 (1933), $317-328$.

[4] Jakimovski, A., The sequence-to-function analogues to Hausdorff transformations, Bulletin of the Research Council of Israel vol. 8, 1959 (1960).

[5] Kęska, S., A variant of the Hausdorff theorem for multi-index matrices II, Linear Algebra Appl. 327 (2001), 17-26.

[6] Lal, S., Approximation of conjugates of almost Lipschitz functions by matrix Cesàro summability method, Arab. J. Math. Sci. 10 (2) (2004), 54.

[7] Lal, S., Mishra, A., Euler-Hausdorff matrix summability operator and trigonometric approximation of the conjugate of a function belonging to the generalized Lipschitz class, J. Inequal. Appl. (2013), 2013:59.

[8] Privalov, I. I., Sur les fonctions conjuguées, Bull. Soc. Math. France 44 (1916), 100103.

[9] Qureshi, K., On the degree of approximation of function belonging to the Lipschitz class by means of a conjugate series, Indian J. Pure Appl. Math. 12 (9) (1981), 1120-1123.

[10] Rhoades, B. E., Ozkoklu, Kevser, Albayrak, Inci, On the degree of approximation of functions belonging to a Lipschitz class by Hausdorff means of its Fourier series, Appl. Math. Comput. 217 (2011), 6868-6871.

[11] Toeplitz, O., Über allgemeine lineare Mittelbildungen, Prace Matematyczno-Fizyczne 22 (1911), 111-119.

Sergiusz Kęska

Institute of Mathematics and Physics

Faculty of Science

Siedlce University of Natural Sciences and Humanities

ul. 3-go Maja 54

08-110 Siedlce

Poland

e-mail: keska@se.onet.pl

Received May 4, 2015 